Épijournal de Géométrie Algébrique

epiga.episciences.org

Volume 1 (2017), Article Nr. 10

\title{
Wonderful compactifications of Bruhat-Tits buildings
}

\author{
Bertrand Rémy, Amaury Thuillier, and Annette Werner
}

\begin{abstract}
Given a split adjoint semisimple group over a local field, we consider the maximal Satake-Berkovich compactification of the corresponding Euclidean building. We prove that it can be equivariantly identified with the compactification we get by embedding the building in the Berkovich analytic space associated to the wonderful compactification of the group. The construction of this embedding map is achieved over a general non-archimedean complete ground field. The relationship between the structures at infinity, one coming from strata of the wonderful compactification and the other from Bruhat-Tits buildings, is also investigated.
\end{abstract}

Keywords. Algebraic group; wonderful compactification; non-archimedean local field; Bruhat-Tits building; Berkovich geometry

2010 Mathematics Subject Classification. 20G15, 14L15; 14L27; 14L30; 20E42; 51E24; $14 \mathrm{G} 22$

\section{[Français]}

Titre. Compactifications magnifiques des immeubles de Bruhat-Tits

Résumé. Étant donné un groupe adjoint semi-simple déployé sur un corps local, nous considérons la compactification de Satake-Berkovich maximale de l'immeuble euclidien correspondant. Nous prouvons qu'elle peut être identifiée de manière équivariante avec la compactification obtenue en plongeant l'immeuble dans l'espace analytique de Berkovich associé à la compactification magnifique du groupe. La construction de ce plongement est effectuée sur un corps complet non-archimédien général. La relation entre les structures à l'infini, l'une venant des strates de la compactification magnifique et l'autre des immeubles de Bruhat-Tits, est également étudiée.

Received by the Editors on February 10, 2017, and in final form on October 17, 2017.

Accepted on November 22, 2017.

Bertrand Rémy

CMLS, École polytechnique, CNRS, Université Paris-Saclay, F-91128 Palaiseau Cedex

e-mail: bertrand.remy@polytechnique.edu

Amaury Thuillier

Université de Lyon, CNRS, Université Lyon 1, Institut Camille Jordan, 43 boulevard du 11 novembre 1918 , F-69622

Villeurbanne cedex

e-mail: thuillier@math.univ-lyon1.fr

Annette Werner

Institut für Mathematik, Goethe-Universität Frankfurt, Robert-Mayer-Str. 6-8, D-60325 Frankfurt a.M.

e-mail: werner@math.uni-frankfurt.de 


\section{Contents}

1. Wonderful compactifications of algebraic groups $\ldots \ldots \ldots \ldots$

2. Construction of the embedding map $\ldots \ldots \ldots \ldots$

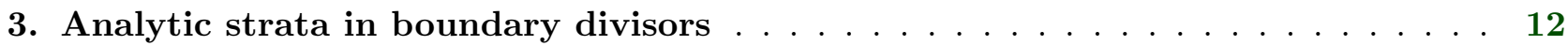

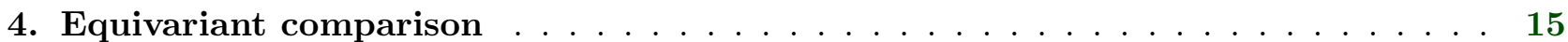

\section{Introduction}

In this paper, we are interested in compactifications of algebraic groups and of some of their related geometries. By "related geometries" we mean "symmetric spaces" and this terminology can be understood in at least two different ways. The first one is purely algebraic and does not require any topological assumption on the ground field: a symmetric space is then the homogeneous space given by the quotient of an adjoint semisimple group by the identity component of the fixed-point set of an involution; the prototype for such a space is $(G \times G) / \operatorname{diag}(G)$ where $\operatorname{diag}(G)$ is the $\operatorname{diag}$ onal $\{(g, g): g \in G\}$. The second meaning makes sense when the ground field is endowed with a complete non-archimedean absolute value; then we investigate a Euclidean building, as given by the Bruhat-Tits theory of reductive groups over valued fields (see [BrT72] and [BrT84]).

To each of the two kinds of symmetric spaces corresponds at least one compactification procedure. The main question of this paper is to understand, when $k$ is a non-archimedean local field, the relationship between the (so-called wonderful) projective variety compactifying the symmetric space $(G \times G) / \operatorname{diag}(G)$ and the Satake-Berkovich compactifications of the associated Bruhat-Tits building $\mathcal{B}(G, k)$, as previously constructed by Berkovich in [Ber90] and by the authors in [RTW10] and [RTW12]. The first space is useful for instance for the algebraic representation theory of the group $G$ while the second one, relevant to the analogy with the Riemannian symmetric spaces of real Lie groups, is useful for the analytic representation theory of, and the harmonic analysis on, the group $G(k)$.

Let us be more precise and consider a split semisimple group of adjoint type $G$ over some field $k$. Wonderful compactifications were initially constructed by representation-theoretic methods (see [CP83], [Str87] and [CS99]) but can now be also constructed by using Hilbert schemes (see [Bri03] and [Bri98]). We adopt the latter viewpoint in the core of the paper, but use the former one in this introduction for simplicity. Let $\rho: G \rightarrow \mathrm{GL}(V)$ be an irreducible representation defined over $k$, assumed to have regular highest weight (strictly speaking, one has to choose a bit more carefully the linear representation $\rho$ in positive characteristic - see [CS99, Lemma 1.7 and Sect. 3]). The projective space $\mathbb{P}(\operatorname{End}(V))$ is a $G \times G$-space for the action defined by: $\left(g, g^{\prime}\right) \cdot M=g M\left(g^{\prime}\right)^{-1}$ for $g, g^{\prime} \in G$ and $M \in \operatorname{End}(V)$. Then the closure $\bar{G}$ of the orbit of $\left[\operatorname{id}_{V}\right]$ is the wonderful compactification of $(G \times G) / \operatorname{diag}(G)$. From the very beginning, it was proved by de Concini and Procesi that the $G \times G$-space $\bar{G}$ is a smooth projective variety containing $(G \times G) / \operatorname{diag}(G)$ as an open orbit and with remarkable geometric properties. For instance (see [CP83]):

- The boundary at infinity $\bar{G} \backslash G$ is a normal crossing divisor whose irreducible components $\left(D_{i}\right)_{i \in I}$ are indexed by the set $I$ of simple roots of the root system of $G$.

- The $G \times G$-orbits are finite in number, their closures are all smooth, in one-to-one correspondence with the subsets of $I$ and there is one single closed orbit.

- Each orbit closure fibers over the product of two flag varieties corresponding to two suitable opposite parabolic subgroups; each fiber is the wonderful compactification of the adjoint semisimple quotient of the intersection of the corresponding parabolics. 
Roughly speaking, $\bar{G}$ does not depend on the chosen representation and its boundary at infinity is not only nice from the viewpoint of algebraic geometry, but also as a Lie-theoretic object; in particular, the appearance of wonderful compactifications of the adjoint semisimple quotients of the various Levi factors contained in $G$ is a beautiful feature of $\bar{G}$.

We assume now that $k$ is a complete non-archimedean valued field and we deal with the Euclidean building $\mathcal{B}(G, k)$ associated to $G$ by Bruhat-Tits theory. In [RTW10], we define a compactification $\overline{\mathcal{B}}_{\tau}(G, k)$ of the building $\mathcal{B}(G, k)$ for each type $\tau$ of parabolic subgroup, and in [RTW12] we show that this finite family of compactifications can be obtained by a suitable analogue of Satake's compactification of Riemmanian symmetric spaces. The compactifications we construct make crucial use of V. Berkovich's approach to analytic non-archimedean geometry; they were in fact originally investigated by Berkovich in [Ber90, Chapter 5] for split groups. This geometry allows one, and actually requires, to use possibly huge complete non-archimedean extensions of $k$; this explains why some of our statements are given for arbitrary complete non-archimedean valued fields, while for a Bruhat-Tits building $\mathcal{B}(G, k)$ to admit a compactification it is necessary and sufficient that $k$ be a local (i.e. locally compact) field. If $k$ is not local, then the topological space $\overline{\mathcal{B}}_{\tau}(G, k)$ is not compact; however, it contains the building $\mathcal{B}(G, k)$ as an open dense subset and the closure of every apartment is compact. Moreover Berkovich theory associates functorially an analytic space (with good local connectedness properties) $X^{\text {an }}$ to any algebraic $k$-variety $X$ in such a way that if $X$ is affine, then $X^{\text {an }}$ can be identified with a suitable set of seminorms on the coordinate ring $k[X]$, and if $X$ is proper then $X^{\text {an }}$ is compact.

In this paper, we only consider the compactification associated to the type of Borel subgroups. It leads to the maximal compactification among those given by the possible types, and we denote it by $\overline{\mathcal{B}}(G, k)$. In [RTW10], the compactification $\overline{\mathcal{B}}(G, k)$ is constructed thanks to the possibility to define an embedding map from $\mathcal{B}(G, k)$ to the Berkovich analytic space associated to the maximal flag variety of $G$. This embedding was constructed first by embedding the building $\mathcal{B}(G, k)$ into the Berkovich space $G^{\text {an }}$, and then by projecting to $\mathcal{F}^{\text {an }}$, where $\mathcal{F}$ is the maximal flag variety of $G$. The outcome is a compactification whose boundary consists of the Bruhat-Tits buildings of all the semisimple quotients of the parabolic $k$-subgroups of $G$ [RTW10, Th. 4.11], a striking similarity with the algebraic case of wonderful compactifications of groups described above.

In order to relate $\overline{\mathcal{B}}(G, k)$ to the wonderful compactification of $G$, a natural idea would be to use the map $\mathcal{B}(G, k) \rightarrow G^{\text {an }}$ (the first step above) and to replace the analytification of the fibration $G \rightarrow \mathcal{F}$ (the second step above) by the analytification of the embedding $G \hookrightarrow \bar{G}, g \mapsto(g, e)$ into the wonderful compactification. However, it turns out that the map $\vartheta: \mathcal{B}(G, k) \rightarrow G^{\text {an }}$ used for compactifying the building is not suitable for this purpose. We have to replace it by a $G(k) \times G(k)$-equivariant map $\Theta: \mathcal{B}(G, k) \times \mathcal{B}(G, k) \rightarrow G^{\text {an }}$ also constructed in [RTW10]. This leads to the desired comparison stated in the following theorem, which is the main goal of this paper.

Theorem. Let $k$ be a complete non-archimedean field and let $G$ be a split adjoint semisimple group over $k$.

(i) There exists a continuous $G(k) \times G(k)$-equivariant map $\bar{\Theta}: \mathcal{B}(G, k) \times \overline{\mathcal{B}}(G, k) \rightarrow \bar{G}^{\text {an }}$. For every point $x$ in $\mathcal{B}(G, k)$ the map $\bar{\Theta}(x,-): \overline{\mathcal{B}}(G, k) \rightarrow \bar{G}^{\text {an }}$ is a $G(k)$-equivariant embedding.

(ii) When $k$ is locally compact, this embedding induces a homeomorphism from the compactified building $\overline{\mathcal{B}}(G, k)$ to the closure of the image of $\mathcal{B}(G, k) \rightarrow G^{\text {an }} \rightarrow \bar{G}^{\text {an }}$.

(iii) The boundaries at infinity are compatible in the following sense: given a proper parabolic $k$ subgroup $P$ of type $\tau(P)$ in $G$, the Bruhat-Tits building of the adjoint semisimple quotient of $P$, which is a stratum of $\overline{\mathcal{B}}(G, k)$, is sent into the analytification of the closed subscheme $\bigcap D_{i}$. 
Part (i) is proven in Theorem 4.1, part (ii) in Proposition 3.1. Part (iii) can be made more precise: it is known that the intersection $\bigcap_{i \notin \tau(P)} D_{i}$ is an orbit closure in the wonderful compactification $\bar{G}$, and that it fibers over $G / P$ with fibers isomorphic to the wonderful compactification of the adjoint semisimple quotient of $P$. Then the Bruhat-Tits building at infinity of the adjoint semisimple quotient of $P$ is sent equivariantly to the analytification of an explicit fiber.

The structure of this paper is as follows. Section 1 recalls the most useful facts for us on wonderful compactifications, adopting Brion's viewpoint using Hilbert schemes of products of a faithful flag variety with itself. Section 2 defines the embedding maps from Bruhat-Tits buildings to analytic spaces associated to wonderful varieties. Section 3 investigates the boundaries of the two compactifications; this is where part (iii) of the theorem above is proved. Section 4 uses the results on the equivariant compatibility of the boundaries to prove the identification between the maximal Satake-Berkovich compactification and the one obtained thanks to analytic wonderful varieties.

Convention. In this paper $G$ is a split adjoint semisimple group over a field $k$. The choice of a maximal split torus $T$ of $G$, with character group $X^{*}(T)$, provides a root system $\Phi(T, G) \subset X^{*}(T)$. In this article, roots are always seen as functions on $T$ and some suitable affine toric varieties associated with $T$.

\section{Wonderful compactifications of algebraic groups}

In this section, we recall the most important facts we need on wonderful compactifications. Our main reference for this topic is Brion's article [Bri03], adopting the viewpoint of Hilbert schemes.

Wonderful compactifications were initially (and are usually) constructed by representation-theoretic means; this was first done over an algebraically closed field of characteristic 0 by de Concini and Procesi [CP83], and then extended by Strickland to the case of positive characteristic [Str87]. Brion's paper establishes, among other things, an identification between the wonderful compactification $\bar{G}$ as in the latter two papers and an irreducible component of the Hilbert scheme $\operatorname{Hilb}(X \times X)$ where $X$ is any suitable flag variety of $G$.

Let us be more precise. Let $k$ be a field and let $G$ be a $k$-split adjoint semisimple group. We choose a parabolic $k$-subgroup $P$ of $G$ such that the $G$-action on the flag variety $X=G / P$ is faithful, which amounts to requiring that $P$ does not contain any simple factor of $G$. As before, we denote by $\bar{G}$ the wonderful compactification obtained via an irreducible representation. The variety $\bar{G}$ admits a $(G \times G)$-action $\left(g, g^{\prime}, \bar{g}\right) \mapsto\left(g, g^{\prime}\right) \cdot \bar{g}$, which we denote by $\left(g, g^{\prime}\right) \cdot \bar{g}=g \bar{g}\left(g^{\prime}\right)^{-1}$ for $g, g^{\prime} \in G$ and $\bar{g} \in \bar{G}$. This notation is motivated by the construction of $\bar{G}$ itself: given a highest weight module $(V, \rho)$ (e.g. obtained as in [CS99, Lemma 1.7]), the compactification $\bar{G}$ is the closure in $\mathbb{P}(\operatorname{End}(V))$ of the $(G \times G)$-orbit of $\left[\operatorname{id}_{V}\right]$ for the action induced by $\left(g, g^{\prime}\right) \cdot M=\rho(g) M \rho\left(g^{\prime}\right)^{-1}$ for any $g, g^{\prime} \in G$ and $M \in \operatorname{End}(V)$.

We now turn specifically to Brion's approach. Let us denote by $\bar{P}$ the closure of $P$ in the complete variety $\bar{G}$ : this space is stable under the restricted action by $P \times P$. Let $\mathcal{G}$ be the space $(G \times G) \times P \times P \bar{P}$ constructed as the image of the quotient map

$$
q: G \times G \times \bar{P} \rightarrow(G \times G) \times{ }^{P \times P} \bar{P}=\mathcal{G}
$$

associated to the right $(P \times P)$-action defined by:

$$
\left(p, p^{\prime}\right) \cdot\left(g, g^{\prime}, \bar{p}\right)=\left(g p, g^{\prime} p^{\prime}, p^{-1} \bar{p} p^{\prime}\right)
$$

for all $g, g^{\prime} \in G, p, p^{\prime} \in P$ and $\bar{p} \in \bar{P}$. The orbit of $\left(g, g^{\prime}, \bar{p}\right)$ for this action is denoted by $\left[g, g^{\prime}, \bar{p}\right]$.

On the one hand, the right $(P \times P)$-action on $G \times G$ is free and the map 


$$
\begin{aligned}
& \pi_{X \times X}: \quad \mathcal{G} \rightarrow X \times X \\
& {\left[g, g^{\prime}, \bar{p}\right] \mapsto\left[g, g^{\prime}\right]=\left(g P, g^{\prime} P\right)}
\end{aligned}
$$

is a locally trivial fibration with fiber $\bar{P}$. On the other hand, the $(G \times G)$-action on $\bar{G}$ restricted to $G \times G \times \bar{P}$ factors through the quotient $q$ to give another map with the same source space as $\pi_{X \times X}$, namely:

$$
\begin{aligned}
\pi_{\bar{G}}: \quad & \mathcal{G} \\
{\left[g, g^{\prime}, \bar{p}\right] } & \mapsto \bar{G} \\
& \mapsto g \bar{p}\left(g^{\prime}\right)^{-1} .
\end{aligned}
$$

By taking the product, one finally obtains a map

$$
\begin{aligned}
& \pi_{X \times X} \times \pi_{\bar{G}}: \quad \mathcal{G} \rightarrow X \times X \times \bar{G} \\
& {\left[g, g^{\prime}, \bar{p}\right] \mapsto\left(g P, g^{\prime} P, g \bar{p}\left(g^{\prime}\right)^{-1}\right)}
\end{aligned}
$$

which is a closed immersion and which enables one to see the fibers of $\pi_{\bar{G}}$ as a flat family of closed subschemes of $X \times X$ (see [Bri03, Sect. 2, p. 610] for more details and additional references to previous work by Brion [Bri98]). The outcome is a $(G \times G)$-equivariant morphism obtained thanks to the universal property of the Hilbert scheme:

$$
\begin{aligned}
\varphi: \bar{G} & \rightarrow \operatorname{Hilb}(X \times X) \\
\bar{g} & \mapsto\left(\pi_{X \times X}\right)_{*}\left(\left(\pi_{\bar{G}}\right)^{*} \bar{g}\right)
\end{aligned}
$$

which, roughly speaking, attaches to any point $\bar{g}$ of the wonderful compactification $\bar{G}$, the following closed subscheme of the product $X \times X$ of faithful flag varieties:

$$
\varphi(\bar{g})=\left\{\left(g P, g^{\prime} P\right) \mid g, g^{\prime} \in G, \bar{g} \in g \bar{P}\left(g^{\prime}\right)^{-1}\right\} \subset X \times X .
$$

This description of the images of $\varphi$ comes from the whole description of the image $\left(\pi_{X \times X} \times \pi_{\bar{G}}\right)(\mathcal{G})$ as an "explicit" incidence variety in $X \times X \times \bar{G}$ [loc. cit.]. It provides an easy way to compute that $\varphi\left(1_{G}\right)$ is the diagonal subscheme $\operatorname{diag}(X)$ in $X \times X$, a point in $\operatorname{Hilb}(X \times X)$ whose stabilizer for the induced $(G \times G)$-action is easily seen to be the diagonal subgroup $\operatorname{diag}(G)$ of $G \times G$. Therefore, using the latter facts together with the $(G \times G)$-equivariance of $\varphi$, one can see $\varphi(\bar{G})$ as the space of degeneracies of the diagonal $\operatorname{diag}(X)$ in $X \times X$, the images of the elements of $G=(G \times G) / \operatorname{diag}(G)$ being the graphs of the elements $g$ seen as automorphisms of $X=G / P$. We will use a more detailed understanding of the boundary points in section 3, but can already quote Brion's comparison theorem [Bri03, Theorem 3]:

Theorem 1.1. Let $\mathcal{H}_{X, G}$ denote the closure of the $(G \times G)$-orbit of $\operatorname{diag}(X)$ in $\operatorname{Hilb}(X \times X)$ endowed with its reduced subscheme structure. Then the map $\varphi$ establishes a $G \times G$-equivariant isomorphism between the wonderful compactification $\bar{G}$ and $\mathcal{H}_{X, G}$.

Note that when $G=\operatorname{Aut}(X)^{\circ}$ (which is the case in general [Dem77]), the space $\mathcal{H}_{X, G}$ is also the irreducible component of $\operatorname{Hilb}(X \times X)$ passing through $\operatorname{diag}(X)$ [Bri03, Lemma 2]. Note also that the above isomorphism holds for any parabolic $k$-subgroup provided the associated flag variety is a faithful $G$-space. The Lie-theoretic construction of the wonderful compactification of $G$ in [Str87], written over an algebraically closed base field of arbitrary characteristic, applies more generally when $G$ is split over an arbitrary field. In [Bri03], Brion always works over an algebraically closed field. However, his construction makes sense over an arbitrary field, and its naturality allows one to see that, by faithfully flat descent, the statement of the above theorem remains true for a split semisimple group $G$ of adjoint type over an arbitrary field $k$.

The proof of this theorem uses a lot of knowledge on the structure of $\bar{G}$, previously obtained by representation theory (see [CP83], [Str87] and [CS99]). In fact, once some standard Lie-theoretic 
choices have been made in $G$, the latter considerations exhibit in the wonderful compactification $\bar{G}$, as a main tool of study of it, an explicit open affine subset $\bar{G}_{0} \subset \bar{G}$. More precisely, let $T$ be a split maximal $k$-torus in $G$ with character group $X^{*}(T)$, let $B_{+}$and $B_{-}$be two opposite Borel subgroups containing $T$ and with unipotent radical $U_{+}$and $U_{-}$, respectively. These choices provide as usual a root system $\Phi=\Phi(T, G) \subset X^{*}(T)$ and two opposite subsets $\Phi_{+}$and $\Phi_{-}$corresponding to the roots appearing in the adjoint $T$-action on the Lie algebras of $U_{+}$and $U_{-}$, respectively. The affine open subset $\bar{G}_{0}$ satisfies the following properties (see for instance [Str87, Section 2] for the original reference in arbitrary characteristic):

- the subset $\bar{G}_{0}$ contains $T$ and is stable under the action by $T U_{-} \times T U_{+}$;

- the closure of $T$ in $\bar{G}_{0}$, which we denote by $Z$, is the affine toric variety associated with the semigroup $\left\langle\Phi^{-}\right\rangle$of $X^{*}(T)$ spanned by the negative roots;

- the canonical map $U_{-} \times Z \times U_{+} \rightarrow \bar{G}$ is an isomorphism onto $\bar{G}_{0}$;

- the subset $Z$ is isomorphic to an affine space of dimension equal to $\operatorname{dim}(T)$, therefore $\bar{G}_{0}$ is isomorphic to an affine space of dimension equal to $\operatorname{dim}(G)$ since $U_{+} \simeq U_{-} \simeq \mathbf{A}_{k}^{d}$, with $2 d=|\Phi|$, as $G$ is split;

- the $(G \times G)$-orbits in $\bar{G}$ are in one-to-one correspondence with the $(T \times T)$-orbits in the toric affine variety $Z$.

In what follows, we see the affine space $Z$ as a partial compactification of the split torus $T \simeq$ $\left(\mathbb{G}_{m}\right)^{\operatorname{dim}(T)}$. Moreover there is a simple way to construct a complete set of representatives of the $(T \times T)$-orbits in $Z$ by "pushing to infinity" the diagonal $\operatorname{diag}(X)$ by suitable one-parameter subgroups in $T$. For instance, given any regular one-parameter subgroup $\lambda: \mathbb{G}_{m} \rightarrow T$, the limit

$$
\lim _{t \rightarrow 0}(\lambda(t), 1) \cdot \operatorname{diag}(X)
$$

exists and is, so to speak, the "most degenerate degeneracy" of the diagonal; it is also the point of $Z$ in the unique closed $(G \times G)$-orbit of $\bar{G}$.

Roughly speaking, the next section, where our embedding map is defined, is the Berkovich analytic counterpart of some of the previous facts.

\section{Construction of the embedding map}

We henceforth assume that the field $k$ is complete with respect to a non-trivial non-archimedean absolute value, and we keep the adjoint split semisimple $k$-group $G$ as before. A non-archimedean field extension of $k$ is a field $K$ containing $k$, which is complete with respect to a non-archimedean absolute value extending the one on $k$.

Our main goal in this section is to construct an equivariant map from the Bruhat-Tits building $\mathcal{B}(G, k)$ to the Berkovich analytic space $\bar{G}^{\text {an }}$ associated to the wonderful compactification $\bar{G}$ of $G$. Note that $\bar{G}^{\text {an }}$ is compact since $\bar{G}$ is proper. More precisely, we define a continuous equivariant map from $\mathcal{B}(G, k) \times \overline{\mathcal{B}}(G, k)$ to $\bar{G}^{\text {an }}$. Fixing a special point in the first coordinate gives the map we aim for. Later, in section 4, we will show that this map is an embedding.

Let us first recall some important facts on Satake-Berkovich compactifications of buildings (see [RTW10] and [RTW12] for details). In [RTW10, Prop. 2.4] we define a morphism $\vartheta: \mathcal{B}(G, k) \rightarrow G^{\text {an }}$ by associating to each point $x$ in the building $\mathcal{B}(G, k)$ a $k$-affinoid subgroup $G_{x}$ of $G^{\text {an }}$ (the underlying set of $G_{x}$ is an affinoid domain of $\left.G^{\text {an }}\right)$. The subgroup $G_{x}$ is an analytic refinement of the integral structure of $G$ associated to $x$ by Bruhat-Tits theory [BrT84, 4.6 and 5.1.30]. Working in an analytic 
context, rather that in a purely algebraic one, has the important advantage that two distinct points, even in the same facet (i.e. the same cell) of $\mathcal{B}(G, k)$, lead to distinct analytic subgroups. We use the group $G_{x}$ to define the image $\vartheta(x)$ as the unique Shilov boundary point of $G_{x}$ [Ber90, 2.4]. The map $\vartheta$ obtained in this way is a continuous $G(k)$-equivariant injection if we let $G(k)$ act on $G^{\text {an }}$ by conjugation [RTW10, Prop. 2.7]. The map $\vartheta$ is well-adapted to Bruhat-Tits theory in the sense that for any non-archimedean extension $K / k$, the group $G_{x}(K)$ is the stabilizer in $G(K)$ of $x$, seen as a point in the building $\mathcal{B}(G, K)$.

Unfortunately, we cannot use the natural map $\vartheta$ to define an embedding towards $\bar{G}^{\text {an }}$ that could be useful for our purposes. We have to use another one, also constructed [loc. cit.] thanks to $\vartheta$. It is a continuous morphism

$$
\Theta: \mathcal{B}(G, k) \times \mathcal{B}(G, k) \rightarrow G^{\text {an }}
$$

which can be seen as a map describing a kind of "relative position" from one point to another. This viewpoint gives an intuition to understand why the equivariance relation

$$
\Theta(g x, h y)=h \Theta(x, y) g^{-1}
$$

is satisfied by $\Theta$ for all $x, y \in \mathcal{B}(G, k)$ and $g, h \in G(k)$ [RTW10, Prop. 2.11]. The definition of $\Theta$ is again an improvement of facts known from Bruhat-Tits theory - here, the transitivity properties of the $G(k)$-action on the facets of $\mathcal{B}(G, k)$ - made possible by the Berkovich analytic viewpoint. Indeed, this viewpoint is flexible enough to allow the use of (possibly huge) non-archimedean extensions of $k$ in order to obtain better transitivity properties. More precisely, for $x, y \in \mathcal{B}(G, k)$ there exists an extension $K / k$ as before and an element $g \in G(K)$ such that after embedding $\mathcal{B}(G, k)$ into $\mathcal{B}(G, K)$ we have $g x=y$. Then we define $\Theta(x, y)$ to be the image of $g \vartheta_{K}(x)$ under the natural projection from $G_{K}^{\text {an }}$ to $G^{\text {an }}$, where $G_{K}$ is the base change of $G$ by $K$, and $\vartheta_{K}: \mathcal{B}(G, K) \rightarrow G_{K}^{\text {an }}$ is the above embedding over $K$. Note that $\Theta$ is compatible with non-archimedean field extensions and that, if $G$ is reductive, we can define the map $\Theta$ on the extended building of $G$ (which then contains the building of the semisimple group $[G, G]$ as a factor). Moreover, by the same Proposition we know that for every point $x_{0}$ in $\mathcal{B}(G, k)$ the map $\Theta\left(x_{0},-\right): \mathcal{B}(G, k) \rightarrow G^{\text {an }}$ is a $G(k)$-equivariant injection, where $G(k)$ acts by left translations on $G^{\text {an }}$.

The key result for our comparison theorem in section 4 is the following statement. It gives a map which, when the first argument is fixed, is eventually shown to be the embedding we are looking for.

Proposition 2.1. The map $\Theta: \mathcal{B}(G, k) \times \mathcal{B}(G, k) \rightarrow G^{\text {an }}$ has a continuous extension

$$
\bar{\Theta}: \mathcal{B}(G, k) \times \overline{\mathcal{B}}(G, k) \rightarrow \bar{G}^{\text {an }},
$$

such that for all $g, h \in G(k), x \in \mathcal{B}(G, k)$ and $y \in \overline{\mathcal{B}}(G, k)$, we have

$$
\bar{\Theta}(g x, h y)=h \bar{\Theta}(x, y) g^{-1} .
$$

The map $\bar{\Theta}$ is compatible with non-archimedean field extensions: if $k^{\prime} / k$ is a non-archimedean extension, then the natural diagram

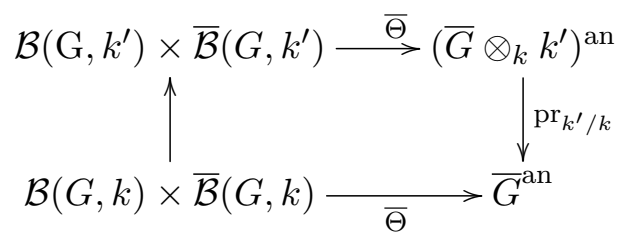

is commutative. 
The rest of the section is dedicated to the proof of this statement. We start with some auxiliary results.

Consider a maximal split torus $T$ of $G$ and a Borel subgroup $B$ of $G$ containing $T$ : such an inclusion $T \subset B$ will henceforth be called a standardization in $G$. We fix a standardization $(T, B)$ in $G$. The Borel group $B$ gives rise to an order on the root system $\Phi=\Phi(T, G)$ inside the character group $X^{*}(T)$ of $T$, and we denote the corresponding set of positive (resp. negative) roots by $\Phi^{+}$(resp. $\Phi^{-}$). Moreover, we denote the associated unipotent subgroups by $U_{+}=\prod_{\alpha \in \Phi^{+}} U_{\alpha}$ and $U_{-}=\prod_{\alpha \in \Phi^{-}} U_{\alpha}$; they are the unipotent radicals of $B$ and of its opposite with respect to $T$, respectively.

We denote by $A$ the apartment associated to $T$ in $\mathcal{B}(G, k)$ : by Bruhat-Tits theory, it is an affine space under the real vector space $X_{*}(T) \otimes_{\mathbf{z}} \mathbf{R}$ where $X_{*}(T)$ is the cocharacter group of $T$, but in accordance with [RTW10] we will see it as an affine space under $\Lambda=\operatorname{Hom}_{\mathbf{A b}}\left(X^{*}(T), \mathbf{R}_{>0}\right)$, using the multiplicative convention for the sake of compatibility with later seminorm considerations. Now, we pick a special point $x_{0}$ in $A$ and we consider the associated épinglage [BrT84, 3.2.1-3.2.2]: this is a consistent choice of coordinates $\xi_{\alpha}: U_{\alpha} \stackrel{\sim}{\rightarrow} \mathbb{G}_{a, k}$ for each root $\alpha$, which identifies the filtration of the root group $U_{\alpha}$ with the canonical filtration of $\mathbb{G}_{a, k}$. Thus we get an isomorphism between the big cell $U_{-} \times T \times U_{+}$and the spectrum of the $k$-algebra $k\left[X^{*}(T)\right]\left[\left(\xi_{\alpha}\right)_{\alpha \in \Phi}\right]$. We also use $x_{0}$ to identify the apartment $A$ with $\Lambda$ : thus there is a natural pairing $\langle$,$\rangle between A$ and $X^{*}(T)$, which we can restrict to a pairing between $A$ and $\Phi$.

At last, we recall that the underlying space of the analytic space associated to an affine $k$-variety $V$ is the set of multiplicative seminorms $k[V] \rightarrow \mathbf{R}$, defined on the coordinate ring of $V$ and extending the absolute value of $k$. Therefore a point in the analytic big cell $\left(U_{-} \times T \times U_{+}\right)^{\text {an }}$ is a multiplicative seminorm on the $k$-algebra $k\left[X^{*}(T)\right]\left[\left(\xi_{\alpha}\right)_{\alpha \in \Phi}\right]$.

We first show an explicit formula for the restriction of $\Theta$ to $A \times A$.

Lemma 2.2. We use the notation introduced above. For each $(x, y) \in A \times A$, the point $\Theta(x, y)$, a priori in $G^{\text {an }}$, actually lies in $\left(U_{-} \times T \times U_{+}\right)^{\text {an }}$. It is given by the following multiplicative seminorm on the coordinate ring $k\left[X^{*}(T)\right]\left[\left(\xi_{\alpha}\right)_{\alpha \in \Phi}\right]$ of $U_{-} \times T \times U_{+}$:

$$
\sum_{\chi \in X^{*}(T), \nu \in \mathbf{N}^{\Phi}} a_{\chi, \nu} \chi \xi^{\nu} \mapsto \max _{\chi, \nu}\left|a_{\chi, \nu}\right|\langle y, \chi\rangle\langle x, \chi\rangle^{-1} \prod_{\alpha \in \Phi_{-}}\langle y, \alpha\rangle^{\nu(\alpha)} \prod_{\alpha \in \Phi_{+}}\langle x, \alpha\rangle^{\nu(\alpha)} .
$$

Note that the seminorm $\Theta(x, y)$ is in fact a norm.

Proof. To check this formula, we first observe that $\Theta\left(x_{0}, x_{0}\right)=\vartheta\left(x_{0}\right)$, so that the desired formula for $\Theta\left(x_{0}, x_{0}\right)$ follows from [RTW10, Prop. 2.6]. Given $(x, y) \in A \times A$, there exist a non-archimedean field extension $K / k$ and points $s, t \in T(K)$ such that we have $\langle x, \chi\rangle=|\chi(t)|$ and $\langle y, \chi\rangle=|\chi(s)|$ for any $\chi \in X^{*}(T)$. By compatibility of $\Theta$ with non-archimedean field extensions and $G(K) \times G(K)$ equivariance [RTW10, Prop. 2.11], we can write $\Theta(x, y)=s \Theta\left(x_{0}, x_{0}\right) t^{-1}$. Since

$$
\chi\left(s w t^{-1}\right)=\chi(s) \chi(t)^{-1} \chi(w) \text { and } \xi_{\alpha}\left(s v s^{-1}\right)=\alpha(s) \xi_{\alpha}(v)
$$

we deduce, for $f=\sum_{\chi, \nu} a_{\chi, \nu} \chi \xi^{\nu} \in k\left[X^{*}(T)\right]\left[\left(\xi_{\alpha}\right)_{\alpha \in \Phi}\right]$, that

$$
\begin{aligned}
|f|(\Theta(x, y)) & =|f|\left(s \Theta\left(x_{0}, x_{0}\right) t^{-1}\right) \\
& =\left|\sum_{\chi, \nu} a_{\chi, \nu} \chi(s) \chi(t)^{-1} \prod_{\alpha \in \Phi_{-}} \alpha(s)^{\nu(\alpha)} \prod_{\alpha \in \Phi_{+}} \alpha(t)^{\nu(\alpha)} \chi \xi^{\nu}\right|\left(\Theta\left(x_{0}, x_{0}\right)\right) \\
& =\max _{\chi, \nu}\left|a_{\chi, \nu}\right|\langle y, \chi\rangle\langle x, \chi\rangle^{-1} \prod_{\alpha \in \Phi_{-}}\langle y, \alpha\rangle^{\nu(\alpha)} \prod_{\alpha \in \Phi_{+}}\langle x, \alpha\rangle^{\nu(\alpha)} .
\end{aligned}
$$

This finishes the proof. 
Let us define a partial compactification of the vector space $\Lambda=\operatorname{Hom}_{\mathbf{A b}}\left(X^{*}(T), \mathbf{R}_{>0}\right)$ by embedding it into

$$
\bar{\Lambda}^{B}=\operatorname{Hom}_{\mathbf{M o n}}\left(\left\langle\Phi^{-}\right\rangle, \mathbf{R}_{\geqslant 0}\right),
$$

where $\left\langle\Phi^{-}\right\rangle$denotes the semigroup spanned by $\Phi^{-}=-\Phi^{+}$in $X^{*}(T)$. The affine space $A$ directed by $\Lambda$ admits a canonical $\Lambda$-equivariant compactification $\bar{A}^{B}$ which can be defined as a contracted product :

$$
\bar{A}^{B}=\bar{\Lambda}^{B} \times{ }^{\Lambda} A=\left(\bar{\Lambda}^{B} \times A\right) / \operatorname{diag}(\Lambda) .
$$

The next step is now, for each standardization $(T, B)$, to use the previous formula in order to extend $\left.\Theta\right|_{A \times A}$ to a continuous map $\bar{\Theta}_{(T, B)}: A \times \bar{A}^{B} \rightarrow \bar{G}^{\text {an }}$.

For this, we need to provide additional details about wonderful compactifications, in particular about the affine charts given by partially compactifying the maximal torus, seen as a factor of the big cell. More precisely, we use the affine subvariety $\bar{G}_{0} \simeq U_{-} \times Z \times U_{+}$of $\bar{G}$ introduced in section 1 . The difference between the latter variety and the big cell is that the factor $T \simeq\left(\mathbb{G}_{m}\right)^{\operatorname{dim}(T)}$ is replaced by a partial compactification $Z$ which is an affine space of dimension equal to $\operatorname{dim}(T)$. At the level of coordinate rings, it means replacing the $k$-algebra $k\left[X^{*}(T)\right]\left[\left(\xi_{\alpha}\right)_{\alpha \in \Phi}\right]$ of the big cell, by the $k$-algebra $k\left[\left\langle\Phi^{-}\right\rangle\right]\left[\left(\xi_{\alpha}\right)_{\alpha \in \Phi}\right]$ of $\bar{G}_{0}$.

Proposition 2.3. Fix a standardization $T \subset B$ of $G$ with associated apartment $A$ and partial compactification $\bar{A}^{B}$. Then the restriction $\left.\Theta\right|_{A \times A}$ extends to a continuous embedding

$$
\bar{\Theta}_{(T, B)}: A \times \bar{A}^{B} \rightarrow \bar{G}^{\text {an }} .
$$

The map $\bar{\Theta}_{(T, B)}$ actually takes its values in $\left(\bar{G}_{0}\right)^{\text {an }}$.

Proof. We use again $x_{0}$ to identify $A$ with $\operatorname{Hom}_{\mathbf{A b}}\left(X^{*}(T), \mathbf{R}_{>0}\right)$ and $\bar{A}^{B}$ with $\operatorname{Hom}_{\mathbf{M o n}}\left(\left\langle\Phi^{-}\right\rangle, \mathbf{R}_{\geqslant 0}\right)$. Thanks to the formula for the restriction of $\Theta$ to $A \times A$ proven in Lemma 2.2, we can easily extend this map to a continuous map $\bar{\Theta}_{(T, B)}: A \times \bar{A}^{B} \rightarrow \bar{G}^{\text {an }}$ by mapping $(x, y) \in A \times \bar{A}^{B}$ to the multiplicative seminorm on the coordinate ring $k\left[\left\langle\Phi^{-}\right\rangle\right]\left[\left(\xi_{\alpha}\right)_{\alpha \in \Phi}\right]$ of $\bar{G}_{0}$ defined by

$$
\sum_{\chi \in\left\langle\Phi^{-}\right\rangle, \nu \in \mathbf{N}^{\Phi}} a_{\chi, \nu} \chi \xi^{\nu} \mapsto \max _{\chi, \nu}\left|a_{\chi, \nu}\right|\langle y, \chi\rangle\langle x, \chi\rangle^{-1} \prod_{\alpha \in \Phi_{-}}\langle y, \alpha\rangle^{\nu(\alpha)} \prod_{\alpha \in \Phi_{+}}\langle x, \alpha\rangle^{\nu(\alpha)} .
$$

The right hand side is obviously continuous in $x$ and $y$, hence $\bar{\Theta}$ is continuous.

Moreover we have

$$
\langle x, \alpha\rangle=\left|\xi_{\alpha}\right|\left(\bar{\Theta}_{(T, B)}(x, y)\right)^{-1}
$$

for each root $\alpha \in \Phi^{+}$and

$$
\langle y, \alpha\rangle=\left|\xi_{\alpha}\right|\left(\bar{\Theta}_{(T, B)}(x, y)\right)
$$

for each root $\alpha \in \Phi^{-}$. Since $\Phi^{+}$spans $X^{*}(T)$, we thus can recover $x$ and $y$ from $\bar{\Theta}_{(T, B)}(x, y)$ and therefore $\bar{\Theta}_{(T, B)}$ is injective.

Remark 2.4. We recall here that, according to [RTW10, Prop. 4.20, (i)], given any pair $(x, y) \in$ $\mathcal{B}(G, k) \times \overline{\mathcal{B}}(G, k)$, there exists a standardization $(T, B)$ such that $(x, y) \in A \times \bar{A}^{B}$ for the apartment $A$ given by $T$. In other words, any $(x, y) \in \mathcal{B}(G, k) \times \overline{\mathcal{B}}(G, k)$ lies in the domain of at least one map $\Theta_{(T, B)}$. 
Our next task is to verify that the extensions $\Theta_{(T, B)}$ glue nicely together when the standardization $(T, B)$ varies, in order to be able to define the map $\bar{\Theta}$ we seek for.

Let us recall some facts about the compactification $\overline{\mathcal{B}}(G, k)$. First, we explain the relationship between the closure of an apartment $A$ in the maximal Satake-Berkovich compactification $\overline{\mathcal{B}}(G, k)$ and the partial compactification $\bar{A}^{B}$ we used so far in this section. We introduce the maximal flag variety $\mathcal{F}=G / B$ of $G$ (where $B$ is some Borel subgroup of $G$ ), and we let $\lambda: G \rightarrow \mathcal{F}$ be the corresponding projection. Then the map $\vartheta_{\varnothing}=\lambda^{\text {an }} \circ \vartheta: \mathcal{B}(G, k) \rightarrow \mathcal{F}^{\text {an }}$ is a $G(k)$-equivariant injection [RTW10, Prop. 3.29]. Let $A$ be an apartment in $G$ associated to the split torus $T$. We denote by $\bar{A}$ the closure of $\vartheta_{\varnothing}(A)$ in $\mathcal{F}^{\text {an: }}$ this is a compact topological space. By [RTW10, Prop. 3.35], the subset $\bar{A}$ is homeomorphic to the compactification of $A$ with respect to the Weyl fan, i.e. the fan consisting of the cones

$$
\mathfrak{C}(P)=\{x \in A: \alpha(x) \leqslant 1 \text { for all } \alpha \in-\Phi(T, P)\},
$$

where $P$ runs over all parabolic subgroups in $G$ containing $T$. The partial compactification $\bar{A}^{B}$ of the present paper is a subset of $\bar{A}$, where only the cone $\mathfrak{C}(B)$ is compactified.

The space $\overline{\mathcal{B}}(G, k)$ is defined as the image of the map

$$
G(k) \times \bar{A} \rightarrow \mathcal{F}^{\text {an }}, \quad(g, x) \mapsto g x g^{-1}
$$

endowed with the quotient topology. If the field $k$ is locally compact, then $\overline{\mathcal{B}}(G, k)$ is the closure of the image of $\mathcal{B}(G, k)$ in $\mathcal{F}^{\text {an }}$ via $\vartheta_{\varnothing}$ and hence compact [RTW10, Prop. 3.34]. At last, the space $\overline{\mathcal{B}}(G, k)$ is the disjoint union of all $\mathcal{B}\left(P_{s s}, k\right)$, where $P$ runs over all parabolic subgroups of $G$, and where $P_{s s}$ denotes the semisimplification $P / R(P)$ of $P$ [RTW10, Th. 4.1].

Lemma 2.5. Let $x$ be a point in $\overline{\mathcal{B}}(G, k)$. For any two apartments $A$ and $A^{\prime}$ of $\mathcal{B}(G, k)$ whose closure in $\overline{\mathcal{B}}(G, k)$ contains $x$, there exists a sequence of points in $A \cap A^{\prime}$ which converges to $x$.

Proof. The stabilizer $G_{x}(k)$ of $x$ in $G(k)$ acts transitively on the set of compactified apartments containing $x$ [RTW10, Prop. 4.20 (ii)], hence we can write $A^{\prime}=g . A$ with $g \in G_{x}(k)$. Pick a standardization $(T, B)$ such that $A=A(T)$ and $x$ belongs to $\bar{A}^{B}$ (cf. Remark 2.4). The assertion is trivially true if $x$ belongs to $\mathcal{B}(G, k)$, hence we may assume that $x$ lies at the boundary of $A$. Then there exists a proper parabolic subgroup $P$ of $G$ containing $B$ such that $x$ lies in the boundary stratum $\mathcal{B}\left(P_{s s}, k\right)$ of $\overline{\mathcal{B}}(G, k)$.

We let $N$ denote the normalizer of $T$ in $G$ and recall that $\Phi=\Phi(T, G)$. By [RTW10, Th. 4.14], the group $G_{x}(k)$ is generated by the stabilizer $N(k)_{x}$ of $x$ in $N(k)$, the full root groups $U_{\alpha}(k)$ when the root $\alpha$ belongs to $\Phi\left(T, R_{u}(P)\right)$, and the partial root groups $U_{\alpha}(k)_{-} \log \alpha(x)$ for $\alpha \in \Phi(T, L)$, where $L$ is the Levi subgroup of $P$ containing $Z_{G}(T)=T$. The group $N(k)$ acts on $A$ by reflections through root hyperplanes, i.e. affine hyperplanes parallel to a linear hyperplane of the form $\{u \mid\langle u, \alpha\rangle=1\}$ in the vector space $\Lambda=\operatorname{Hom}\left(X^{*}(T), \mathbf{R}_{>0}\right)$, with $\alpha \in \Phi$. Identifying $A$ and $\Lambda$, it follows that the group $N(k)_{x}$ fixes each point of the closure $\bar{A}_{x}^{B}$ of the affine subspace

$$
A_{x}=\left\{y \in A \mid \alpha(y)=\alpha(x) \text { for all } \alpha \in \Phi^{-} \text {such that } \alpha(x) \neq 0\right\} .
$$

Consider a root $\alpha \in \Phi$ and an element $u$ in $U_{\alpha}(k)$. The action of $u$ on $\mathcal{B}(G, k)$ fixes each point of the half-space

$$
A_{u}=\left\{y \in A|\alpha(y) \geqslant| \xi_{\alpha}(u) \mid\right\} .
$$

The closure of the latter in $\bar{A}^{B}$ is the subspace $\bar{A}_{u}^{B}$ defined by

$$
\bar{A}_{u}^{B}=\left\{y \in \bar{A}^{B}|\alpha(y) \geqslant| \xi_{\alpha}(u) \mid\right\} \text { if } \alpha \in \Phi^{-}
$$


and

$$
\bar{A}_{u}^{B}=\left\{y \in \bar{A}^{B} \quad|(-\alpha)(y)| \xi_{\alpha}(u) \mid \leqslant 1\right\} \text { if } \alpha \in \Phi^{+} .
$$

In each case, if $\bar{A}_{u}^{B}$ contains $x$, then $\bar{A}_{u}^{B} \cap \bar{A}_{x}^{B}$ is a neighborhood of $x$ in $\bar{A}_{x}^{B}$.

Now, thanks to the description of $G_{x}(k)$ recalled above, any given element $g$ of $G_{x}(k)$ fixes each point in the intersection of $\bar{A}_{x}^{B}$ with a finite number of (partially) compactified half-spaces $\bar{A}_{u}^{B}$ all containing $x$, hence fixes each point in some neighborhood $V$ of $x$ in $\bar{A}_{x}^{B}$. We deduce $V \subset \bar{A}^{B} \cap g \bar{A}^{B}$ and, therefore, there exists a sequence of points in $A \cap g A$ which converges to $x$ in both $\bar{A}^{B}$ and $g \bar{A}^{B}$.

Proof of Proposition 2.1. We are now in position to prove successively the properties claimed about the map $\bar{\Theta}$.

1) Existence. We first check that the maps $\bar{\Theta}_{(T, B)}$ glue together nicely. Pick two points $x \in \mathcal{B}(G, k)$ and $y \in \overline{\mathcal{B}}(G, k)$. We have to check that

$$
\bar{\Theta}_{(T, B)}(x, y)=\bar{\Theta}_{\left(T^{\prime}, B^{\prime}\right)}(x, y)
$$

for any two standardizations $(T, B)$ and $\left(T^{\prime}, B^{\prime}\right)$ such that $\overline{A(T)}^{B}$ and ${\overline{A\left(T^{\prime}\right)}}^{B^{\prime}}$ both contain $x$ and $y$. By Lemma 2.5, we can pick a sequence $\left(y_{n}\right)_{n \geqslant 0}$ in $A(T) \cap A\left(T^{\prime}\right)$ converging to $y$ in $\overline{A(T)}{ }^{B}$ and $\overline{A\left(T^{\prime}\right)} B^{\prime}$. We have

$$
\bar{\Theta}_{(T, B)}\left(x, y_{n}\right)=\Theta\left(x, y_{n}\right)=\bar{\Theta}_{\left(T^{\prime}, B^{\prime}\right)}\left(x, y_{n}\right)
$$

for all $n$, hence $\bar{\Theta}_{(T, B)}(x, y)=\bar{\Theta}_{\left(T^{\prime}, B^{\prime}\right)}(x, y)$ by continuity of $\bar{\Theta}_{(T, B)}$ and $\bar{\Theta}_{\left(T^{\prime}, B^{\prime}\right)}$.

Since any two points $x \in \mathcal{B}(G, k)$ and $y \in \overline{\mathcal{B}}(G, k)$ are contained in $\overline{A(T)}$ B for a suitable standardization $(T, B)$ (cf. Remark 2.4), this allows us to define the map $\bar{\Theta}$ by gluing together the maps $\bar{\Theta}_{(T, B)}$.

2) Equivariance. We now check that the map $\bar{\Theta}$ is $G(k) \times G(k)$-equivariant, and for this we pick $(x, y) \in \mathcal{B}(G, k) \times \overline{\mathcal{B}}(G, k)$ and choose a standardization $(T, B)$ such that the partially compactified apartment $\bar{A}^{B}$ for $A=A(T)$ contains both $x$ and $y$, as well as a sequence $\left(y_{n}\right)_{n \geqslant 0}$ in $A$ converging to $y$. By the Bruhat decomposition theorem for compactifed buildings, proved in [RTW10, Prop. 4.20], we can write $G(k)=G_{x}(k) N G_{x}(k)$, where $G_{x}(k)=\operatorname{Stab}_{G(k)}(x)$ and $N=\operatorname{Norm}_{G}(T)(k)$. Therefore, it is enough to prove that

$$
\bar{\Theta}(g x, y)=\bar{\Theta}(x, y) g^{-1} \text { and } \bar{\Theta}(x, h y)=h \bar{\Theta}(x, y)
$$

for $g$ and $h$ belonging to $G_{x}(k)$ or $N$. If $g \in N$, then $g x \in A$ and therefore

$$
\begin{aligned}
\bar{\Theta}(g x, y) & =\bar{\Theta}_{(T, B)}(g x, y)=\lim _{n} \Theta\left(g x, y_{n}\right)=\lim _{n} \Theta\left(x, y_{n}\right) g^{-1} \\
& =\bar{\Theta}_{(T, B)}(x, y) g^{-1}=\bar{\Theta}(x, y) g^{-1} .
\end{aligned}
$$

If $g \in G_{x}(k)$, then

$$
\begin{aligned}
\bar{\Theta}(g x, y) & =\bar{\Theta}(x, y)=\bar{\Theta}_{(T, B)}(x, y) \\
& =\lim _{n} \Theta\left(x, y_{n}\right)=\lim _{n} \Theta\left(g x, y_{n}\right)=\lim _{n} \Theta\left(x, y_{n}\right) g^{-1} \\
& =\bar{\Theta}_{(T, B)}(x, y) g^{-1}=\bar{\Theta}(x, y) g^{-1}
\end{aligned}
$$

If $h \in N$, then $h \bar{A}^{B}=\bar{A}^{h B h^{-1}}$ and

$$
\begin{aligned}
\bar{\Theta}(x, h y) & =\bar{\Theta}_{\left(T, h B h^{-1}\right)}(x, h y)=\lim _{n} \Theta\left(x, h y_{n}\right)=\lim _{n} h \Theta\left(x, y_{n}\right) \\
& =h \bar{\Theta}_{(T, B)}(x, y)=h \bar{\Theta}(x, y) .
\end{aligned}
$$


If $h \in G_{x}(k)$, then the points $x=h x$ and $h y_{n}$ are contained in the apartment $h A$ and therefore

$$
\begin{aligned}
\bar{\Theta}(x, h y) & =\bar{\Theta}_{\left(h T h^{-1}, h B h^{-1}\right)}(x, h y)=\lim _{n} \Theta\left(x, h y_{n}\right)=\lim _{n} h \Theta\left(x, y_{n}\right) \\
& =h \bar{\Theta}_{(T, B)}(x, y)=h \bar{\Theta}(x, y) .
\end{aligned}
$$

3) Continuity. Let us now prove continuity of $\bar{\Theta}$. The canonical map

$$
(G(k) \times G(k)) \times\left(A \times \bar{A}^{B}\right) \rightarrow \mathcal{B}(G, k) \times \overline{\mathcal{B}}(G, k)
$$

identifies the right-hand-side with a topological quotient of the left-hand-side. By construction and equivariance, the map $\bar{\Theta}$ is induced by the continuous map

$$
(G(k) \times G(k)) \times\left(A \times \bar{A}^{B}\right) \rightarrow \bar{G}^{\text {an }}, \quad((g, h),(x, y)) \mapsto h \bar{\Theta}_{(T, B)}(x, y) g^{-1},
$$

hence it is continuous.

4) Field extensions. Finally, the map $\bar{\Theta}$ is compatible with non-archimedean field extensions since this is the case for each map $\bar{\Theta}_{(T, B)}$ thanks to the formula used to define it in the proof of Lemma 2.2 .

\section{Analytic strata in boundary divisors}

In this section, we analyze the compatibility between the boundaries at infinity of the Satake-Berkovich compactifications of Bruhat-Tits buildings and of the wonderful compactifications. For this, we need to recall some facts about the combinatorics and geometry of boundaries of wonderful compactifications $\bar{G}$, which amounts to decomposing the latter varieties into $G \times G$-orbits. Our general reference is [Bri03, Section 3, p. 617].

Let $\operatorname{Par}(G)$ be the scheme of parabolic subgroups of $G$. The type $\tau=\tau(P)$ of a parabolic subgroup $P$ of $G$ is the connected component of $\operatorname{Par}(G)$ containing $P$; we denote by $\operatorname{Par}_{\tau}(G)$ this connected component. Since $G$ is split, each connected component of $\operatorname{Par}(G)$ contains a $k$-rational point. Let $\mathcal{T}=\pi_{0}(\operatorname{Par}(G))$ denote the set of types of parabolic subgroups. This set is partially ordered as follows: given two types $\tau$ and $\tau^{\prime}$, we set $\tau \leqslant \tau^{\prime}$ if there exist $P \in \operatorname{Par}_{\tau}(G)(k)$ and $P^{\prime} \in \operatorname{Par}_{\tau^{\prime}}(G)(k)$ with $P \subset P^{\prime}$. The minimal type corresponds to Borel subgroups and the maximal type corresponds to the trivial parabolic subgroup $G$. This set is also equipped with an involution $\tau \mapsto \tau^{\text {opp }}$ defined as follows: pick a parabolic subgroup $P \in \operatorname{Par}_{\tau}(G)(k)$ as well as a Levi subgroup $L$ of $P$ and set $\tau^{\text {opp }}=\tau\left(P^{\text {opp }}\right)$, where $\mathrm{P}^{\text {opp }}$ is the only parabolic subgroup of $G$ such that $P \cap \mathrm{P}^{\text {opp }}=L$. Note that the type $\tau^{\text {opp }}$ is well-defined since $G(k)$ acts transitively by conjugation on pairs $(P, L)$ consisting of a parabolic subgroup of type $\tau$ and a Levi subgroup $L$ of $P$.

Let us go back now to the problem of decomposing $\bar{G}$ as explicitly as possible into $G \times G$-orbits. We pick a standardization $(T, B)$ of $G$ and use the associated notation as in the previous section, such as the root system $\Phi$ and its positive and negative subsets $\Phi^{+}$and $\Phi^{-}$. We let also $\Delta \subset \Phi_{-}$denote the corresponding set of simple roots and we recall that there is an increasing one-to-one correspondence between the types of parabolics introduced above and the subsets of $\Delta$ : this map sends the type $\tau$ of a parabolic subgroup $P$ containing $B$ to $\Delta \cap \Phi(T, L)$, where $L$ is the Levi subgroup of $P$ containing $T$; in particular, the type of Borel subgroups (resp. of the trivial subgroup $G$ ) goes to $\varnothing$ (resp. to $\Delta$ ). The choice of $(T, B)$ gives us the "partially compactified big cell" $\bar{G}_{0}$, which can be identified with $U_{-} \times Z \times U_{+}[\operatorname{Str} 87$, Lemmas 2.1 and 2.2] via the natural open immersion

$$
\varphi: U_{-} \times Z \times U_{+} \rightarrow \bar{G}_{0}, \quad\left(u_{-}, z, u_{+}\right) \mapsto u_{-} z u_{+}^{-1} .
$$


One can choose specific 1-parameter subgroups $\lambda_{\tau}: \mathbf{G}_{m} \rightarrow T$ defined by $\alpha\left(\lambda_{\tau}\right)(t)=1$ if $\alpha \in \tau$ and $\alpha\left(\lambda_{\tau}\right)(t)=t$ if $\alpha \in \Delta \backslash \tau$.

Each of these cocharacters has a limit at 0 , i.e. extends to a morphism from $\mathbf{A}_{k}^{1}$ to $Z$. We set

$$
e_{(T, B), \tau}=\lim _{t \rightarrow 0} \lambda_{\tau}(t) \in Z(k)
$$

and we note that this $k$-rational point can be described by

$$
\alpha\left(e_{(T, B), \tau}\right)=0 \text { for all } \alpha \in-\Phi\left(T, R_{u}(P)\right) \quad \text { and } \quad \alpha\left(e_{(T, B), \tau}\right)=1 \text { for all } \alpha \in \Phi(T, L)^{-} .
$$

The points $\left\{e_{(T, B), \tau}\right\}_{\tau \subset \Delta}$ are extremely useful because they provide a complete set of representatives:

- for the $T \times T$-action on the toric affine variety $Z$,

- for the $G \times G$-action on the wonderful compactification $\bar{G}$.

Therefore we obtain a one-to-one correspondence between these two sets of orbits.

Let $P$ denote the unique parabolic subgroup of $G$ of type $\tau$ containing $B$ (it can be described as consisting of the elements $g \in G$ such that the limit $\lambda_{\tau}(t) g \lambda_{\tau}(t)^{-1}$ exists as $\left.t \rightarrow 0\right)$, and let $L$ be its Levi subgroup containing $T=Z_{G}(T)$. Then $P^{\text {opp }}$ is the parabolic subgroup in $G$ opposite $P$ with respect to $B$ (it can be described as consisting of the elements $g \in G$ such that the limit $\lambda_{\tau}(t) g \lambda_{\tau}(t)^{-1}$ exists as $t \rightarrow \infty$, and we have $\left.P \cap P^{\text {opp }}=L\right)$. We have the following description of stabilizers:

$$
\operatorname{Stab}_{G \times G}\left(e_{(T, B), \tau}\right)=\operatorname{diag}(L)\left(R_{u}(P) Z(L) \times R_{u}\left(P^{\mathrm{opp}}\right) Z(L)\right) \subset P \times P^{\mathrm{opp}} .
$$

In other words, the wonderful compactification $\bar{G}$ has a $G \times G$-equivariant stratification by locally closed subspaces $\{X(\tau)\}_{\tau \in \mathcal{T}}$ and each stratum $X(\tau)$ is a homogeneous space under $G \times G$ which comes with a $G \times G$-equivariant map

$$
\pi_{\tau}: X(\tau) \rightarrow \operatorname{Par}_{\tau}(G) \times \operatorname{Par}_{\tau^{\text {opp }}}(G)
$$

sending the point $e_{(T, B), \tau}$ to $\left(P, P^{\text {opp }}\right)$ (note that this map is well-defined since the stabilizer of $e_{(T, B), \tau}$ is contained in $\left.P \times P^{\text {opp }}\right)$. Moreover, for each point $\left(P, P^{\prime}\right) \in \operatorname{Par}_{\tau}(G)(k) \times \operatorname{Par}_{\tau^{\text {opp }}}(G)(k)$ consisting of two opposite parabolic subgroups with respect to a common Levi subgroup $L$, the fiber of $\pi_{\tau}$ over $\left(P, P^{\prime}\right)$ is canonically isomorphic to the adjoint quotient $L / Z(L)$ of $L$.

One can also give an explicit description of the intersection of $X(\tau)$ with $\bar{G}_{0}$ and of the restriction of $\pi_{\tau}$ to $X(\tau) \cap \bar{G}_{0}$. For simplicity, let us write $\Phi(Q)=\Phi(T, Q)$ for every subgroup $Q$ of $G$ containing the torus $T$. The stratum $X(\tau)$ intersects the toric variety $Z=\operatorname{Speck}\left[\left\langle\Phi^{-}\right\rangle\right]$along the locally closed subspace

$$
Z(\tau)=\left\{z \in Z: \alpha(z)=0 \text { for all } \alpha \in-\Phi\left(R_{u}(P)\right) \text { and } \alpha(z) \neq 0 \text { for all } \alpha \in \Phi(L)^{-}\right\},
$$

i.e. the intersection of the vanishing sets of all negative roots belonging to the unipotent radical of $P^{\text {opp }}$ and the non-vanishing set of all negative roots belonging to the Levi subgroup $L$. This stratum $Z(\tau)$ is a principal homogeneous space under $T / T(\tau)$, where $T(\tau)$ is the subtorus given as the connected component of the kernel of all $\alpha \in \Phi(L)$, and $Z(\tau)$ is trivialized by the $k$-rational point $e_{(T, B), \tau}$. The torus $T(\tau)$ is the center of $L$, hence $T / T(\tau)$ is the maximal split torus of $L / Z(L)$ induced by $T$.

At last, the following diagram

$$
\begin{gathered}
\prod_{\alpha \in-\Phi\left(R_{u}(P)\right)} U_{\alpha} \times\left(\prod_{\alpha \in \Phi(L)^{-}} U_{\alpha} \times Z(\tau) \times \prod_{\alpha \in \Phi(L)^{+}} U_{\alpha}\right) \times \prod_{\alpha \in \Phi\left(R_{u}(P)\right)} U_{\alpha} \stackrel{\varphi}{\longrightarrow} X(\tau) \cap \bar{G}_{0} \\
\prod_{\alpha \in-\Phi\left(R_{u}(P)\right)} U_{\alpha} \times \prod_{\alpha \in \Phi\left(R_{u}(P)\right)} U_{\alpha}
\end{gathered}
$$


where the bottom horizontal map is $\left(u_{-}, u_{+}\right) \mapsto\left(u_{-} P u_{-}^{-1}, u_{+} P^{\mathrm{opp}} u_{+}^{-1}\right)$, is commutative.

Let $P$ be a parabolic subgroup of $G$ of type $\tau$, and let $\lambda: G \rightarrow G / P$ be the projection to the associated flag variety, which is isomorphic to $\operatorname{Par}_{\tau}(G)$. Recall the embedding $\vartheta: \mathcal{B}(G, k) \rightarrow G^{\text {an }}$ defined in [RTW10, Prop. 2.4]. By composition, we get a map $\vartheta_{\tau}=\lambda^{\text {an }} \circ \vartheta: \mathcal{B}(G, k) \rightarrow \operatorname{Par}_{\tau}(G)^{\text {an }}$, which is $G(k)$-equivariant and independent of the choice of the parabolic $P$ of type $\tau$ by [RTW10, Lemma 2.13]. If $\tau$ is the type of a Borel subgroup, we have seen this map under the name $\vartheta_{\emptyset}$ already in section 2.

Proposition 3.1. Let $P$ be any parabolic subgroup of $G$ of type $\tau(\neq \Delta)$, giving rise to the boundary stratum $\mathcal{B}\left(P_{\text {ss }}, k\right)$ lying in $\overline{\mathcal{B}}(G, k) \backslash \mathcal{B}(G, k)$.

(i) The map $\bar{\Theta}$ sends $\mathcal{B}(G, k) \times \mathcal{B}\left(P_{\text {ss }}, k\right)$ into $X(\tau)^{\text {an }}$.

(ii) We have

$$
\left(\pi_{\tau}^{\text {an }} \circ \bar{\Theta}\right)(x, y)=\left(P, \vartheta_{\tau^{\text {opp }}}(x)\right)
$$

for all $(x, y) \in \mathcal{B}(G, k) \times \mathcal{B}\left(P_{s s}, k\right)$.

(iii) For every point $x \in \mathcal{B}(G, k)$, the restriction of $\bar{\Theta}(x, \cdot)$ to $\mathcal{B}\left(P_{s s}, k\right)$ is a continuous embedding.

Remark 3.2. Note that in assertion (ii) above, $P$ is a $k$-rational point in $\operatorname{Par}_{\tau}(G)^{\text {an }}$ whereas the point $\vartheta_{\tau^{\text {opp }}}(x)$ in $\operatorname{Par}_{\tau^{\text {opp }}}(G)^{\text {an }}$ is defined over a transcendental non-archimedean field extension. One should also be aware that, if one denotes by $|X|$ the underlying topological space of a non-Archimedean analytic space $X$, then $|X \times Y|$ is in general different from $|X| \times|Y|$. However, since $X(k) \times|Y| \subset$ $|X \times Y|$, the formula in point (ii) does make sense.

Proof of Proposition 3.1. We fix a standardization $(T, B)$ of $G$ and use the notation introduced above.

Let us prove (i) and (ii). The partially compactified apartment $\bar{A}^{B}$ intersects $\mathcal{B}\left(P_{s s}, k\right)$ along the subspace $\bar{A}^{B}(P)$ defined by the conditions $\alpha=0$ for each root $\alpha$ in $-\Phi\left(R_{u}(P)\right)$ and $\alpha>0$ for each root $\alpha \in \Phi(L)^{-}$. This is the apartment of the maximal split torus $T / T(\tau)$ of $P_{s s}$. According to the explicit formula for $\bar{\Theta}_{(T, B)}$ in Proposition 2.3, a point $(x, y) \in A \times \bar{A}^{B}(P)$ is mapped to the Gauss point of

$$
\left(\prod_{\alpha \in-\Phi\left(R_{u}(P)\right)} U_{\alpha} \times\left(\prod_{\alpha \in \Phi(L)^{-}} U_{\alpha} \times Z \times \prod_{\alpha \in \Phi(L)^{+}} U_{\alpha}\right) \times \prod_{\alpha \in \Phi\left(R_{u}(P)\right)} U_{\alpha}\right)^{\text {an }}
$$

defined by

$$
|\alpha|=\langle y, \alpha\rangle\langle x, \alpha\rangle^{-1} \text { for all } \alpha \in \Phi^{-},
$$

which vanishes if and only if $\alpha \in-\Phi\left(R_{u}(P)\right)$, and by

$$
\left|\xi_{\alpha}\right|= \begin{cases}0 & \text { if } \alpha \in-\Phi\left(R_{u}(P)\right) \\ \langle y, \alpha\rangle & \text { if } \alpha \in \Phi(L)^{-} \\ \langle x, \alpha\rangle & \text { if } \alpha \in \Phi(L)^{+} \cup \Phi\left(R_{u}(P)\right) .\end{cases}
$$

By the commutative diagram preceding our statement, we thus have $\bar{\Theta}_{(B, T)}(x, y) \in\left(X(\tau) \cap \bar{G}_{0}\right)^{\text {an }}$. Using the explicit formula for $\vartheta_{\tau^{\text {opp }}}(x)$ from the proof of [RTW10, Lemma 3.33], we also find that $\pi_{\tau}^{\text {an }}\left(\bar{\Theta}_{(T, B)}(x, y)\right)$ is the point $\left(\{P\}, \vartheta_{\tau^{\text {opp }}}(x)\right)$ of $\operatorname{Par}_{\tau}(G)^{\text {an }} \times \operatorname{Par}_{\tau^{\text {opp }}}(G)^{\text {an }}$. We have thus proved (i) and (ii) for the restriction of $\bar{\Theta}$ to $A \times \bar{A}^{B}(P)$. Since $\pi_{\tau}$ is $G \times G$-equivariant, the general case follows via translation by the subgroup $G \times P$.

Let us finally prove (iii). Consider two points $y, y^{\prime}$ in $\mathcal{B}\left(P_{s s}, k\right)$ such that $\bar{\Theta}(x, y)=\bar{\Theta}\left(x, y^{\prime}\right)$. Pick a maximal split torus $T$ of $G$ contained in $P$ such that $y$ and $y^{\prime}$ belong to the closure of $A=A(T)$; for 
any choice of a Borel subgroup $B$ of $P$ containing $T$, we have $y, y^{\prime} \in \bar{A}^{B}$. Choose also some $g \in G(k)$ such that $x$ belongs to $g^{-1} A$. By assumption, we have

$$
\bar{\Theta}(g x, y)=\bar{\Theta}(x, y) g^{-1}=\bar{\Theta}\left(x, y^{\prime}\right) g^{-1}=\bar{\Theta}\left(g x, y^{\prime}\right) .
$$

Since $g x, y$ and $y^{\prime}$ are all contained in $\bar{A}^{B}$, we deduce

$$
\bar{\Theta}_{(T, B)}(g x, y)=\bar{\Theta}_{(T, B)}\left(g x, y^{\prime}\right)
$$

and therefore $y=y^{\prime}$ by injectivity of $\bar{\Theta}_{(T, B)}(g x,-)$ on $\bar{A}^{B}$.

\section{Equivariant comparison}

Thanks to the better understanding of the relationship between the boundaries provided by the previous section, we can now prove our main comparison theorem, stated as the first two points of the main theorem in the introduction.

Theorem 4.1. (i) For every point $x \in \mathcal{B}(G, k)$, the map

$$
\bar{\Theta}(x, \cdot): \overline{\mathcal{B}}(G, k) \rightarrow \bar{G}^{\text {an }}
$$

is a continuous, $G(k)$-equivariant embedding.

(ii) Assume that $k$ is locally compact. Then $\bar{\Theta}(x, \cdot)$ is a closed embedding, and the compactified building $\overline{\mathcal{B}}(G, k)$ is homeomorphic to the closure of the image of the building $\mathcal{B}(G, k)$ under the embedding

$$
\mathcal{B}(G, k) \stackrel{\Theta(x, \cdot)}{\longrightarrow} G^{\text {an }} \hookrightarrow \bar{G}^{\text {an }} .
$$

Proof. Injectivity of $\bar{\Theta}(x, \cdot)$ follows immediately from the following three observations based on Proposition 3.1. For any parabolic subgroup $P$ of $G$ of type $\tau$ :

- the map $\bar{\Theta}(x, \cdot)$ sends $\mathcal{B}\left(P_{s s}, k\right)$ into $X(\tau)^{\text {an }}$;

- the image of $\bar{\Theta}\left(x, \mathcal{B}\left(P_{s s}, k\right)\right)$ under the map $\pi_{\tau}$ is contained into $\{P\} \times \operatorname{Par}_{\tau(P)}{ }^{\text {opp }}(G)^{\text {an }}$;

- the map $\bar{\Theta}(x, \cdot)$ restricts injectively to the stratum $\mathcal{B}\left(P_{s s}, k\right)$ of $\overline{\mathcal{B}}(G, k)$ associated with $P$.

Note that, thanks to the first two observations, different strata have disjoint images.

If $k$ is locally compact, the map $\bar{\Theta}(x, \cdot)$ is closed since it is continuous, $\overline{\mathcal{B}}(G, k)$ is compact by [RTW10, Prop. 3.34], and $\bar{G}^{\text {an }}$ is Hausdorff. Since $\mathcal{B}(G, k)$ is dense in $\overline{\mathcal{B}}(G, k)$ by [RTW10, Prop. 3.34], the last claim follows.

In order to complete the proof of our main theorem stated in the introduction, it remains to show part (iii). Strictly speaking, this statement deals with orbits closures while Proposition 3.1 deals with the orbits themselves. The relationship is in fact very neat since it is well-known that, with our notation, we have by [CS99, Th. 3.9]:

$$
\overline{X(\tau)}=\bigcap_{i \in \Delta \backslash \tau} D_{i}
$$

hence

$$
\overline{X(\tau)}=\bigcup_{\tau^{\prime} \leqslant \tau} X\left(\tau^{\prime}\right)
$$


By Prop. 3.1 (ii), this observation implies that, for every parabolic subgroup $P$ of type $\tau$,

$$
\mathcal{B}\left(P_{s s}, k\right) \subset X(\tau)^{\text {an }} \text { and } \overline{\mathcal{B}}\left(P_{s s}, k\right) \subset \overline{X(\tau)}^{\text {an }}
$$

since

$$
\overline{\mathcal{B}}\left(P_{s s}, k\right)=\bigcup_{P^{\prime} \in \operatorname{Par}(G)(k), P^{\prime} \subset P} \mathcal{B}\left(P_{s s}^{\prime}, k\right)
$$

by [RTW10, Theorem 4.1 and Example 3.9.(ii)].

We conclude by a strengthening of assertion (i) in our Theorem, thereby answering a question raised by one of the referees.

Proposition 4.2. The map

$$
\bar{\Theta}: \mathcal{B}(G, k) \times \overline{\mathcal{B}}(G, k) \rightarrow \bar{G}^{\text {an }}
$$

is injective.

Proof. (Step 1) Let us first check that the map $\Theta: \mathcal{B}(G, k) \times \mathcal{B}(G, k) \rightarrow G^{\text {an }}$ is injective, a fact which we did not notice in [RTW10]. Consider two points $(x, y),\left(x^{\prime}, y^{\prime}\right) \in \mathcal{B}(G, k) \times \mathcal{B}(G, k)$ such that $\Theta(x, y)=\Theta\left(x^{\prime}, y^{\prime}\right)$. By [RTW10, Lemma 2.10], this condition still holds after an arbitrary nonArchimedean extension of $k$ and therefore we may assume that there exists $g^{\prime}, h, h^{\prime} \in G(k)$ such that $x^{\prime}=g^{\prime} x, y=h x$ and $y^{\prime}=h^{\prime} x$. By [RTW10, Proposition 2.11], we get

$$
h \Theta(x, x)=h^{\prime} \Theta(x, x) g^{\prime-1},
$$

hence

$$
h^{-1} h^{\prime} \vartheta(x)=\vartheta(x) g^{\prime} \quad \text { and } \quad h^{-1} h^{\prime} G_{x}=G_{x} g^{\prime}
$$

by [RTW10, Definition 2.9 and Proposition 2.4.(i)]. We thus can write $g^{\prime}=h^{-1} h^{\prime} s$ for some suitable $s \in G_{x}(k)$ and deduce

$$
g^{\prime} G_{x}=h^{-1} h^{\prime} G_{x}=G_{x} g^{\prime}, \text { hence } G_{g^{\prime} \cdot x}=G_{x} .
$$

This implies $g^{\prime} \cdot x=x$ by [RTW10, Corollary 2.5], and therefore $g^{\prime} \in G_{x}(k)$. It follows that $h^{-1} h^{\prime}$ also belongs to $G_{x}(k)$, hence $x^{\prime}=g^{\prime} x=x$ and $y^{\prime}=h^{\prime} x=h\left(h^{-1} h^{\prime}\right) x=h x=y$.

(Step 2) Let us now prove that $\bar{\Theta}$ is also injective. It follows easily from Proposition 3.1 (i) and (ii) that the map $\bar{\Theta}$ separates the strata $\mathcal{B}(G, k) \times \mathcal{B}\left(P_{s s}, k\right)$ associated with the parabolic subgroups $P$ of $G$, so it is enough to check that its restriction to each stratum $\mathcal{B}(G, k) \times \mathcal{B}\left(P_{s s}, k\right)$ is injective. We remark that it is easy if the parabolic subgroup $P$ does not contain any simple factor of $G$, i.e. if its type $\tau=\tau(P)$ is nondegenerate. In this case indeed, the opposite type $\tau^{\text {opp }}$ is also nondegenerate, hence the map $\vartheta_{\tau^{\text {opp }}}$ from $\mathcal{B}(G, k)$ to $\operatorname{Par}_{\tau^{\text {opp }}}(G)^{\text {an }}$ is injective [RTW10, Proposition 3.29] and thus the conclusion follows from Proposition 3.1.(ii) and (iii).

(Step 3) In general, one can write $G=G_{1} \times G_{2}$ for some semisimple groups of adjoint type $G_{1}$ and $G_{2}$ such that $P=G_{1} \times P_{2}$, where $P_{2}$ is a nondegenerate parabolic subgroup of $G_{2}$ of type $\tau_{2}$. The schemes $\operatorname{Par}_{\tau}(G) \times \operatorname{Par}_{\tau^{\text {opp }}}(G)$ and $\operatorname{Par}_{\tau_{2}}\left(G_{2}\right) \times \operatorname{Par}_{\tau_{2}^{\text {opp }}}\left(G_{2}\right)$ are canonically isomorphic, the building of $G$ is the product of the buildings of $G_{1}$ and $G_{2}$, and $\vartheta_{\tau}=\vartheta_{\tau_{2}} \circ p r_{2}$. Fix a point $x_{2}$ in $\mathcal{B}\left(G_{2}, k\right)$. By Proposition 3.1(ii), the restriction of $\bar{\Theta}$ to

$$
\mathcal{B}\left(G_{1}, k\right) \times\left\{x_{2}\right\} \times \mathcal{B}\left(P_{s s}, k\right)=\mathcal{B}\left(G_{1}, k\right) \times\left\{x_{2}\right\} \times \mathcal{B}\left(G_{1}, k\right) \times \mathcal{B}\left(P_{2, s s}, k\right)
$$

is a $G_{1} \times P_{s s}$-equivariant map whose image is contained in the fiber of $\pi_{\tau}$ over the point $\left(P, \vartheta_{\tau_{2}}\left(x_{2}\right)\right)$, which is a canonically split torsor under $G_{1}^{\text {an }} \times\left(P_{2, s s} / Z\left(P_{2, s s}\right)\right)^{\text {an }}$. Projecting onto $\mathrm{G}_{1}^{\text {an }}$, we thus obtain a $G_{1} \times G_{1}$-equivariant map

$$
M: \mathcal{B}\left(G_{1}, k\right) \times \mathcal{B}\left(G_{1}, k\right) \times \mathcal{B}\left(P_{2, s s}, k\right) \rightarrow\left(G_{1} \otimes_{k} K\right)^{\text {an }},
$$


where $K$ is equal to the completed residue field $\mathcal{H}\left(\vartheta_{\tau_{2}}\left(x_{2}\right)\right)$. Choosing a standardization $(B, T)=$ $\left(B_{1} \times B_{2}, T_{1} \times T_{2}\right)$ of $G$ and computing in the associated big cell as in the proof of Proposition 3.1, we observe that the restriction of $M$ to

$$
A \times \bar{A}^{B}(P)=A\left(T_{1}\right) \times A\left(T_{2}\right) \times A\left(T_{1}\right) \times \bar{A}^{B_{2}}\left(P_{2}\right)
$$

factors through the canonical projection onto $A\left(T_{1}\right) \times A\left(T_{1}\right)$, and that the induced map coincides with the restriction of $\Theta_{G_{1} \otimes_{k} K}$ to $A\left(T_{1}\right) \times A\left(T_{1}\right)$. By translation, we deduce that $M$ factors through the projection onto $\mathcal{B}\left(G_{1}, k\right) \times \mathcal{B}\left(G_{1}, k\right)$ and that the induced map to $G_{1, K}^{\text {an }}$ coincides with $\Theta_{G_{1} \otimes_{k} K}$.

(Step 4) We can now finish the proof. We make the identification

$$
\mathcal{B}(G, k) \times \mathcal{B}\left(P_{s s}, k\right)=\mathcal{B}\left(G_{1}, k\right) \times \mathcal{B}\left(G_{2}, k\right) \times \mathcal{B}\left(G_{1}, k\right) \times \mathcal{B}\left(P_{2, s s}, k\right)
$$

and consider two points $\left(x_{1}, x_{2}, y_{1}, y_{2}\right),\left(x_{1}^{\prime}, x_{2}^{\prime}, y_{1}^{\prime}, y_{2}^{\prime}\right)$ in the product such that $\bar{\Theta}\left(\left(x_{1}, x_{2}\right),\left(y_{1}, y_{2}\right)\right)=$ $\bar{\Theta}\left(\left(x_{1}^{\prime}, x_{2}^{\prime}\right),\left(y_{1}^{\prime}, y_{2}^{\prime}\right)\right)$. Projecting this equality by $\pi_{\tau}$, we deduce $\vartheta_{\tau_{2}}\left(x_{2}\right)=\vartheta_{\tau_{2}}\left(x_{2}^{\prime}\right)$, hence $x_{2}^{\prime}=x_{2}$. Combining steps 1 and 3 , we then get $\left(x_{1}^{\prime}, y_{1}^{\prime}\right)=\left(x_{1}, y_{1}\right)$. We can then apply Proposition 3.1.(iii) to derive $\left(y_{1}^{\prime}, y_{2}^{\prime}\right)=\left(y_{1}, y_{2}\right)$, hence $\left(x_{1}^{\prime}, x_{2}^{\prime}, y_{1}^{\prime}, y_{2}^{\prime}\right)=\left(x_{1}, x_{2}, y_{1}, y_{2}\right)$.

Further questions: Our constructions are all Galois-equivariant and can be descended to ground fields over which the group $G$ need not be split. On the one hand, it seems to us that wonderful compactifications of non-split groups are less explicitly described in the literature, presumably due to lack of representation-theoretic motivation. On the other hand, descent in Bruhat-Tits theory is a central topic. The geometric description of Satake-Berkovich compactifications of Bruhat-Tits buildings could be a useful tool to describe wonderful compactifications of non-split groups.

Another interesting line of further research is the generalization of our results to other equivariant compactifications of the reductive group $G$ [Ti03].

Acknowledgements. We thank the anonymous referees for their questions and remarks, which helped us to improve the paper in many ways. The first author is very grateful to the Humboldt Foundation for its support, he would like to thank the participants of the Institut Fourier workshop "Symmetric spaces" held a few years ago and the Institut für Mathematik (FB12) of the GoetheUniversität for his optimal stay in Frankfurt during the Fall Semester of 2017. The second author was partially supported by the GeoLie project of the Agence Nationale de la Recherche (project ANR-15CE40-0012). The third author would like to thank Deutsche Forschungsgemeinschaft for supporting this joint work under grant WE 4279/7.

\section{References}

[Ber90] V.G. Berkovich, Spectral theory and analytic geometry over non-archimedean fields. Mathematical Surveys and Monographs, vol. 33, American Mathematical Society, Providence, 1990. MR-1070709

[Bri98] M. Brion, The behaviour at infinity of the Bruhat decomposition, Comment. Math. Helv. 73 (1998), no. 1, 137-174. MR-1610599

[Bri03] M. Brion, Group completions via Hilbert schemes, J. Algebraic Geom. 12 (2003), no. 4, 605-626. MR-1993758

[BrT72] F. Bruhat and J. Tits, Groupes réductifs sur un corps local, I. Données radicielles valuées, Publ. Math. Inst. Hautes Études Sci. 41 (1972), 5-251. MR-0327923 
[BrT84] F. Bruhat and J. Tits, Groupes réductifs sur un corps local, II. Schémas en groupes. Existence d'une donnée radicielle valuée, Publ. Math. Inst. Hautes Études Sci. 60 (1984), 197-376. MR-756316

[CP83] C. de Concini and C. Procesi, Complete symmetric varieties. In: Invariant theory (Montecatini, 1982), pp. 1-44, Lecture Notes in Math., vol. 996, Springer, 1983. MR-718125

[CS99] C. de Concini and T. A. Springer, Compactification of symmetric varieties, Transformation Groups 4 (1999), no. 2-3, 273-300. MR-1712864

[Dem77] M. Demazure, Automorphismes et déformations des variétés de Borel, Invent. Math. 39 (1977), 179-186. MR-0435092

[EJ08] S. Evens and B.F. Jones, On the wonderful compactification, preprint 2008. arXiv: 0801.0456

[RTW10] B. Rémy, A. Thuillier, and A. Werner, Bruhat-Tits theory from Berkovich's point of view I. Realizations and compactifications of buildings, Ann. Sci. Éc. Norm. Supér. (4) 43 (2010), no. 3, 461-554. MR-2667022

[RTW12] B. Rémy, A. Thuillier, and A. Werner, Bruhat-Tits theory from Berkovich's point of view II. Satake compactifications of buildings, J. Inst. Math. Jussieu 11 (2012), no. 2, 421-465. MR-2905310

[Str87] E. Strickland, A vanishing theorem for group compactifications, Math. Ann. 277 (1987), no. 1, 165-171. MR-884653

[Ti03] D.A. Timashev, Equivariant compactifications of reductive groups, Sb. Math. 194 (2003), no. 3-4, 589-616. MR-1992080 